\title{
Variation in premaxillary tooth count and a developmental abnormality in a tyrannosaurid dinosaur
}

Tetsuto Miyashita, Darren H. Tanke, and Philip J. Currie

Acta Palaeontologica Polonica 55 (4), 2010: 635-643 doi: http://dx.doi.org/10.4202/app.2009.0067

Premaxillary tooth count tends to be stable amongst toothed dinosaurs, and most theropods have four teeth in each premaxilla. Only one case of bilaterally asymmetric variation is known in theropod premaxillary dentition, and there is no record of ontogenetic or individual variation in premaxillary tooth count. Based on these observations, a tyrannosaurid left premaxilla with three teeth (TMP 2007.20.124) is an interesting deviation and represents an unusual individual of Daspletosaurus sp. with a developmental abnormality. The lower number of teeth is coupled with relatively larger alveoli, each of which is capable of hosting a larger than normal tooth. This indicates that tooth size and dental count vary inversely, and instances of reduction in tooth count may arise from selection for increased tooth size. On the other hand, the conservative number of premaxillary teeth in most theropods implies strong developmental constraints and a functional trade-off between the dimensions of the premaxillary alveolar margin and the size of the teeth. In light of recent advances in the study of tooth morphogenesis, tooth count is a function of two parameters: dimensions of an odontogenic field for a tooth series, and dimensions of tooth positions. A probable developmental cause for the low tooth count of TMP 2007.20.124 is that the dimensions of the alveoli expanded by approximately a third during tooth morphogenesis. Numerical traits such as tooth count are difficult to treat in a phylogenetic analysis. When formulating a phylogenetic character, a potential alternative to simply counting is to rely on the morphological signature for developmental parameters that control the number of the element in question.

Key words: Dinosauria, Theropoda, Tyrannosauridae, tooth count, developmental regulation, Dinosaur Park Formation, Alberta, Canada.

Tetsuto Miyashita [tetsuto@ualberta.ca] and Philip J. Currie [Philip.Currie@ualberta.ca ], Department of Biological Sciences, University of Alberta, Edmonton, AB, T6E 2G9 Canada; Darren H. Tanke [Darren.Tanke@gov.ab.ca], Royal Tyrrell Museum of Palaeontology, Drumheller, AB, Canada. 
This is an open-access article distributed under the terms of the Creative Commons

Attribution License (for details please see creativecommons.org), which permits unrestricted use, distribution, and reproduction in any medium, provided the original author and source are credited.

FoF 